\title{
Following the Electrons: Simulation for High-Resolution STEM and CBED
}

Mark P. Oxley ${ }^{1,2 *}$

${ }^{1}$ Center for Nanophase Materials Sciences, Oak Ridge National Laboratory, Oak Ridge, TN, USA.

2. Institute for Functional Imaging of Materials, Oak Ridge National Laboratory, Oak Ridge, TN, USA.

* Corresponding author: oxleymp@ornl.gov

Image simulation plays an important role in the interpretation of high-resolution scanning transmission electron microscopy (STEM) images and spectroscopy. STEM images are often used as a starting point for structural calculations based on density functional theory (DFT). It is only by comparison of detailed simulation with high-quality experiment that we can confirm the results of these calculations. This is particularly true for localized structures such as interfaces, defects, and grain boundaries. For example, low level doping can lead to structural distortions that result in contrast changes in mediumangle annular dark field (ADF) STEM images. The use of simulation allows us to differentiate between image contrasts due to small structural changes and other possible causes such as surface contamination. Simulation of electron energy loss spectroscopy (EELS) intensities can be a useful tool for determining the abruptness of interfaces or local vacancy structures. In addition, recent developments in pixelated electron detectors allows the acquisition of scanning diffraction patterns. The role of simulation in the interpretation of these data sets will be discussed.

There are many high-quality electron image simulation packages available that are primarily based on the frozen phonon (Fph)/quantum excitation of phonons (QEP) models [1, 2]. These multislice approaches provide a robust platform for image simulation when used correctly. There are, however, a number of potential traps for using these packages, which can result in simulations that are not truly quantitative. Primary among these is the issue of convergence and how it affects simulations of different STEM imaging modes. This tutorial is not intended to provide a detailed discussion of the physics underlying these approaches but rather to offer a practical guide to image simulation and how to ensure a meaningful result. While the examples in this tutorial are simulated using the $\mu$ STEM package, the concepts discussed are generally applicable [3].

The starting point for most simulations is an experimental result. Can you explain the image that you have acquired? This is also the point where one must decide if there is enough information to do a meaningful simulation. In other words, is the experiment sufficiently well-defined to obtain a definitive statement about the sample from the simulation. It is important to be able to correctly characterize the electron probe and detectors. It is not simply the alignment and the size of the detectors but also the determination of the black level settings, i.e., do we know what zero signal reads as? In addition, a reasonable estimate of the specimen thickness is required.

Having determined the details of the sample and the experiment, the next item to be determined is the size of the real space supercell and the number of pixels to include in the mesh. For simplicity we will consider a square unit cell. The supercell is formed by tiling the unit cell and an overall size of approximately $30 \AA$ is usually adequate. The maximum range in reciprocal space is determined by the Nyquist frequency, $k_{\max }= \pm 1 / 2 \Delta x$, where $\Delta x$ is the real space pixel size. In practice this range is reduced further by applying a band width limit (BWL). This arrangement is illustrated in Figure 1. The BWL sets the maximum Fourier component of the incident electrons wave function. The value of these 
parameters will affect not only the quality of the simulation, but the amount of memory required and the calculation time. The judicious choice of these parameters will be discussed in detail.

The Fph and QEP algorithms both proceed by using multiple atomic configurations generated by jiggling the atoms within the supercell to account for lattice vibrations. The probe is then propagated through different configurations (phase grates) several times in order to provide a statistically meaningful measure of the atomic positions. This raises the question of how many configurations are required and how many times the probe should be propagated through the model structure. How do you determine if your calculation is converged? The number of phase grates and passes required depends very much on the model (monolayer vs. bulk crystal) and the detector (high-angle ADF vs. CBED). Strategies for checking convergence will be discussed.

In summary, there are many steps involved in performing a useful, quantitative STEM simulation. Many parameters need to be examined on a case-by-case basis and sometimes compromises must be made to make a simulation practical with the computing resources at hand. It is the goal of this tutorial to outline the steps to a successful calculation that can be published with confidence [4].

References:

[1] RF Loane, P Xu and J Silcox, Acta. Cryst. A 47 (1991), p. 267.

[2] BD Forbes et al., Phys. Rev. B 82, (2010), 104103.

[3] LJ Allen, AJ D’Alfonso and SD Findlay, Ultramicroscopy 151 (2015), p. 11.

[4] Research supported by the U.S. Department of Energy, Office of Basic Energy Sciences, Materials Sciences and Engineering Division. This manuscript has been authored by UT-Battelle, LLC, under contract DE-AC05-00OR22725 with the US Department of Energy (DOE). The US government retains and the publisher, by accepting the article for publication, acknowledges that the US government retains a nonexclusive, paid-up, irrevocable, worldwide license to publish or reproduce the published form of this manuscript, or allow others to do so, for US government purposes. DOE will provide public access to these results of federally sponsored research in accordance with the DOE Public Access Plan (http://energy.gov/downloads/doe-public-access-plan).
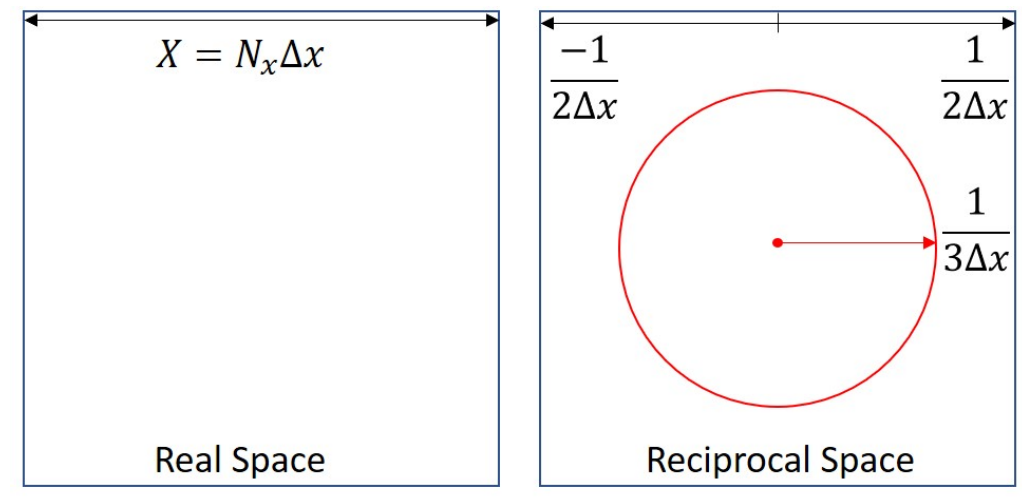

Figure 1. Simple illustration of sizing of square mesh for a multislice simulation showing relationship between real and reciprocal space. Red circle indicates the band width limit. 